\title{
Optimized methods for IL-17A refolding and anti-IL17A Fab production for co-crystallization with small molecules
}

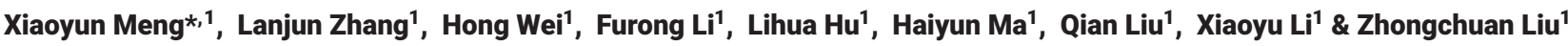 \\ ${ }^{1}$ Discovery Project Unit, HitGen Inc., Chengdu, China; *Author for correspondence: xiaoyun.meng@hitgen.com
}

BioTechniques 69: 71-76 (July 2020) 10.2144/btn-2019-0170

First draft submitted: 27 December 2019; Accepted for publication: 23 March 2020; Published online: 27 April 2020

\begin{abstract}
Refolding of human interleukin 17A (IL-17A) has been reported; however, the key refolding protocol was not robust enough to deliver consistent results and to be easily scaled up for crystallization. Here we report an optimized refolding method for IL-17A. Although co-crystal structures of IL-17A with ligands have been obtained with a high-affinity peptide and an anti-IL-17A Fab as stabilizers, neither the production yield nor the characterization of the IL-17A/Fab complex was reported. To facilitate co-crystallization of IL-17A with small-molecule compounds derived from our DNA encoded library, we also describe the method for yield enhancement of anti-IL-17A Fab production and characterize the IL-17A/Fab complex for the first time, providing an essential prerequisite for structure-based drug discovery targeting IL-17A.
\end{abstract}

\section{METHOD SUMMARY}

An Escherichia coli system was used for IL-17A expression. Two-way optimizations of the refolding conditions were implemented by exploring various ratios of oxidized and reduced forms of the oxido-shuffling reagents and the refolding duration. A detailed protocol for the optimized refolding has been submitted to protocols.io (https://www.protocols.io/view/optimized-refolding-protocol-for-il-17a-bda3i2gn). Yield enhancement of anti-IL-17A Fab production was achieved after generating a stable HEK293-F cell line expressing the Fab fragment by G418 selection of transfected cells. The purified IL-17A/anti-IL-17A Fab complex was characterized by SDS-PAGE and western blotting. Single crystals of the quaternary complex, IL-17A/Fab/HAP (high affinity peptide) with small-molecule compounds derived from our DNA encoded library could be obtained using a batch method.

\section{KEYWORDS:}

co-crystallization $\bullet$ DNA encoded library $\bullet$ Fab $\bullet$ interleukin 17A (IL-17A) • oxido-shuffling reagent $\bullet$ refolding $\bullet$ structure-based drug design

Human interleukin 17A (IL-17A), the prototype of the IL-17 family, is a principal driver of multiple inflammatory and autoimmune diseases [1,2]. It is an attractive target for therapeutic interventions, and its structural and functional properties are of great medical interest. The native human IL-17A, mainly secreted from helper T cells as a glycoprotein, is a homodimer of 34-38 kDa containing six cysteinyl residues [3]. Antibodies that neutralize IL-17A or its receptor (IL-17RA) deliver efficacy in autoimmune diseases, but no small-molecule IL-17A antagonists have yet progressed into clinical trials. To facilitate the drug discovery process, multiple pharmaceutical companies have been actively engaged in structure-based drug design on IL-17A. To date, several groups have published crystal structures of human IL-17A in apo form and various bound forms [4-9]. In their crystallization efforts, one group obtained the recombinant IL-17A through mammalian expression followed by deglycosylation, or through insect expression with truncation and site-directed mutagenesis to suppress glycosylation and eliminate the predicted exposed free thiol group [4,5]. In contrast, other groups chose $E$. coli expression due to its multiple advantages, such as high expression levels, lack of posttranslational modifications and cost-effectiveness [6,10]. A total of four isoforms of a recombinant human IL-17A variant post-refolding were reported, differing by their interchain disulfide structures [11].

To obtain sufficient IL-17A for crystallization, we also chose the E. coli system for expression. A cDNA encoding human IL-17A (20155) without tag, was codon-optimized and synthesized by Genscript, then inserted into Nde I and Hind III sites of a pET30a plasmid (Novagen, Cat\# 69909-3). The expression vector was transformed into E. coli BL21 (DE3), and isolation of inclusion bodies was performed according to the published protocol by Wu et al. [10]. Two protocols for IL-17A refolding have been published previously. One used the oxido-shuffling pair of cysteine and cystamine at $5 \mathrm{mM}$ and $1 \mathrm{mM}$ respectively [10]; the other used another oxido-shuffling pair, reduced and oxidized glutathione, at $0.3 \mathrm{mM}$ and $0.03 \mathrm{mM}$ respectively [6]. Both protocols applied more of the reducing agent than the oxidizing agent (Table 1), which is in accordance with the commonly established conditions for refolding of disulfide-bonded proteins [12-14]. We tested both protocols, but neither was found to be robust enough to deliver consistent results or to be scaled up; therefore we implemented two-way optimizations of the refolding conditions by exploring various ratios of oxidized and reduced forms of the oxido- 


\begin{tabular}{|c|c|c|c|c|}
\hline Refolding condition feature & Protein & Oxido-shuffling pair & Concentrations (mM) & Ref. \\
\hline \multirow[t]{3}{*}{ More reducing agent } & IL-17A & Cysteine, Cystamine & 5,1 & [10] \\
\hline & IL-17A & GSH, GSSG & $0.3,0.03$ & [6] \\
\hline & $\begin{array}{l}\text { Not } \\
\text { specified, disulfide-containing } \\
\text { protein }\end{array}$ & GSH, GSSG & 5,1 & {$[12,13]$} \\
\hline \multirow[t]{2}{*}{ More oxidizing agent } & IL-5 & GSH, GSSG & $0.1,1$ & [15] \\
\hline & IL-5 & GSH, GSSG & $0.2,1$ & [16] \\
\hline Equal or more oxidizing agent & IL-17A & GSH, GSSG & $0.3,0.3 ; 0.2,1$ & \\
\hline
\end{tabular}

The list includes those published for IL-17A, IL-5, etc. and those confirmed to be robust in-house at HitGen Inc. (listed in the bottom row)

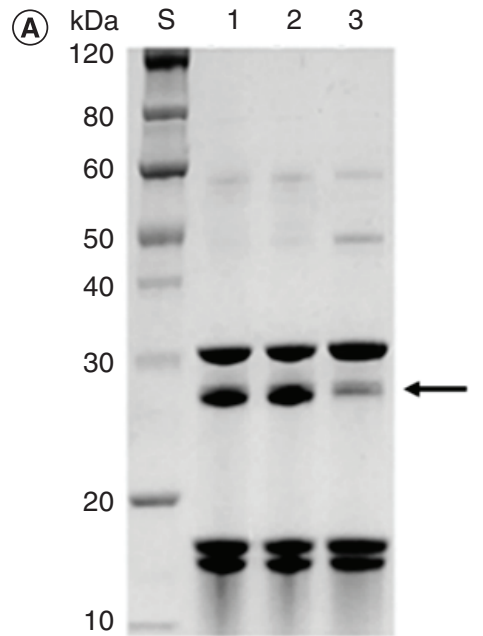

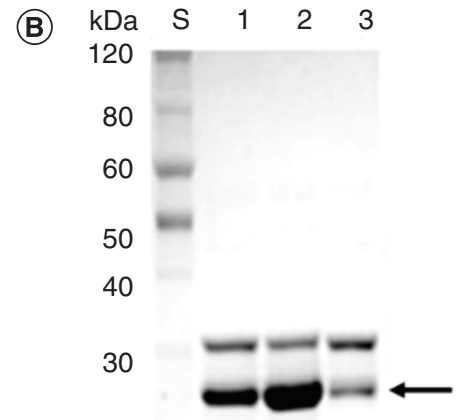

20

10
Figure 1. Effect of different ratios of GSH:GSSG on the refolding efficiency of IL-17A. The refolded protein was purified with SP-FF chromatography [10] before gel loading. (A) Refolding duration: $40 \mathrm{~h}$. (B) refolding duration: $60 \mathrm{~h}$. The three sets of refolding conditions were: 1: GSH:GSSG at a molar ratio of 1:5, with GSH and GSSG at 0.2 and $1 \mathrm{mM}$, respectively; 2: GSH:GSSG at a molar ratio of 1:1, with GSSG and GSH both at $0.3 \mathrm{mM} ; 3$ : GSH:GSSG at a molar ratio of 5:1, with GSH and GSSG at 1 and $0.2 \mathrm{mM}$, respectively. S: Protein standard for SDS-PAGE (PAGE-Master Plus MM1397-500, Genscript).

shuffling reagents and the refolding duration. While in the refolding buffer (100 mM Tris- $\mathrm{HCl}, \mathrm{pH} 8.0,1 \mathrm{M} \mathrm{L}$-arginine), equal or more oxidized glutathione, with GSH and GSSG at a 1:5 molar ratio (0.2 mM GSH and $1 \mathrm{mM} \mathrm{GSSG}$ ), or with GSH and GSSG at 1:1 molar ratio (0.3 mM GSH and $0.3 \mathrm{mM} \mathrm{GSSG}$ ), was the best ratio to produce the correctly refolded IL-17A covalent dimer (Table 1, bottom row; Figure 1, band indicated by arrow). This isoform is the most active of the four, containing two interchain disulfide bonds [11]. As the refolding duration was $40 \mathrm{~h}$, the percentages of this isoform present were similar (25-30\%) with both equal and more GSSG, as shown in Figure 1A (lanes 1 and 2). With an extended refolding duration of around $60 \mathrm{~h}$, the percentages increased to $45 \%$ and $60 \%$, respectively, as shown in Figure $1 \mathrm{~B}$ (lanes 1 and 2). The experiment was performed multiple times with consistent results. In contrast, when more reducing glutathione was added, with GSSG and GSH at 1:5 molar ratio, either at a lower concentration of $1 \mathrm{mM}$ or a higher concentration of $5 \mathrm{mM}$, a much lower percentage ( $\sim 10 \%$ with $40 \mathrm{~h}$ refolding, $15 \%$ with $60 \mathrm{~h}$ refolding) of the correctly folded IL-17A covalent dimer was obtained (Figure $1 \mathrm{~A}$ $\& B$, lane 3). Interestingly, another cytokine, IL-5, could be refolded similarly with more oxidized form than reduced form of glutathione (Table 1), which could be due to the requirement for cysteine residues to form interchain and/or intrachain disulfide bonds to convey maximum bioactivity of the protein $[15,16]$. The optimized refolding method we report here is robust enough to be scaled up to meet the requirement for protein crystallization.

For the purification of the refolded IL-17A after desalting, we initially followed the protocol generated by Wu et al. [10], but later with the gradually improved refolding efficiency, we found that the five chromatographic purification steps could be simplified to only three columns, in the order SP-FF, fluoroapatite and Mono S chromatography; RP and Source 15S chromatography could be removed without compromising the final diffraction quality. Moreover, if refolding was carried out under the optimal conditions (1:1 molar ratio of GSH:GSSG for $60 \mathrm{~h}$ ), Mono S chromatography could also be removed. The details of each chromatographic step were as previously described [10]. The final overall yield was at least $9.3 \mathrm{mg}$ or $6.7 \%$ of the total protein amount obtained from refolding, more than double the reported $3.1 \%$ [10]. The purity of IL-17A was analyzed by $12 \%$ SDS-PAGE (Figure 2A), and by RP-HPLC (Shimazdu LC2030C, Boston Green ODS C18 column, $.4 .6 \mathrm{~mm} \times 150 \mathrm{~mm}, 5 \mu \mathrm{m}$, Boston Analytics, Inc.) to be over 95\% (Figure 2B). The protein activity was analyzed by a cell-based cytokine induction assay using a human CXCL1 assay kit (Cat\# 62HCXC1PEG, Cisbio). The result indicated that our $E$. coli-derived IL-17A exhibited superior bioactivity when compared with commercial ones from various expression systems. The detailed EC50 values are listed in the bottom table of Figure 3.

There has been active research in identifying orally available small-molecule inhibitors of IL-17A despite the challenges of targeting the protein-protein interactions associated with the large, shallow IL-17A/IL-17RA interfaces. Liu et al. investigated a series of linear 


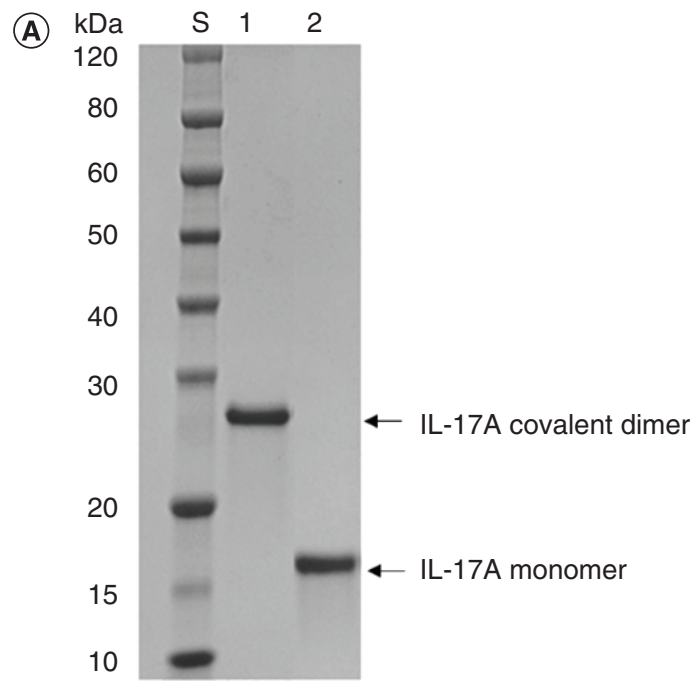

(B)

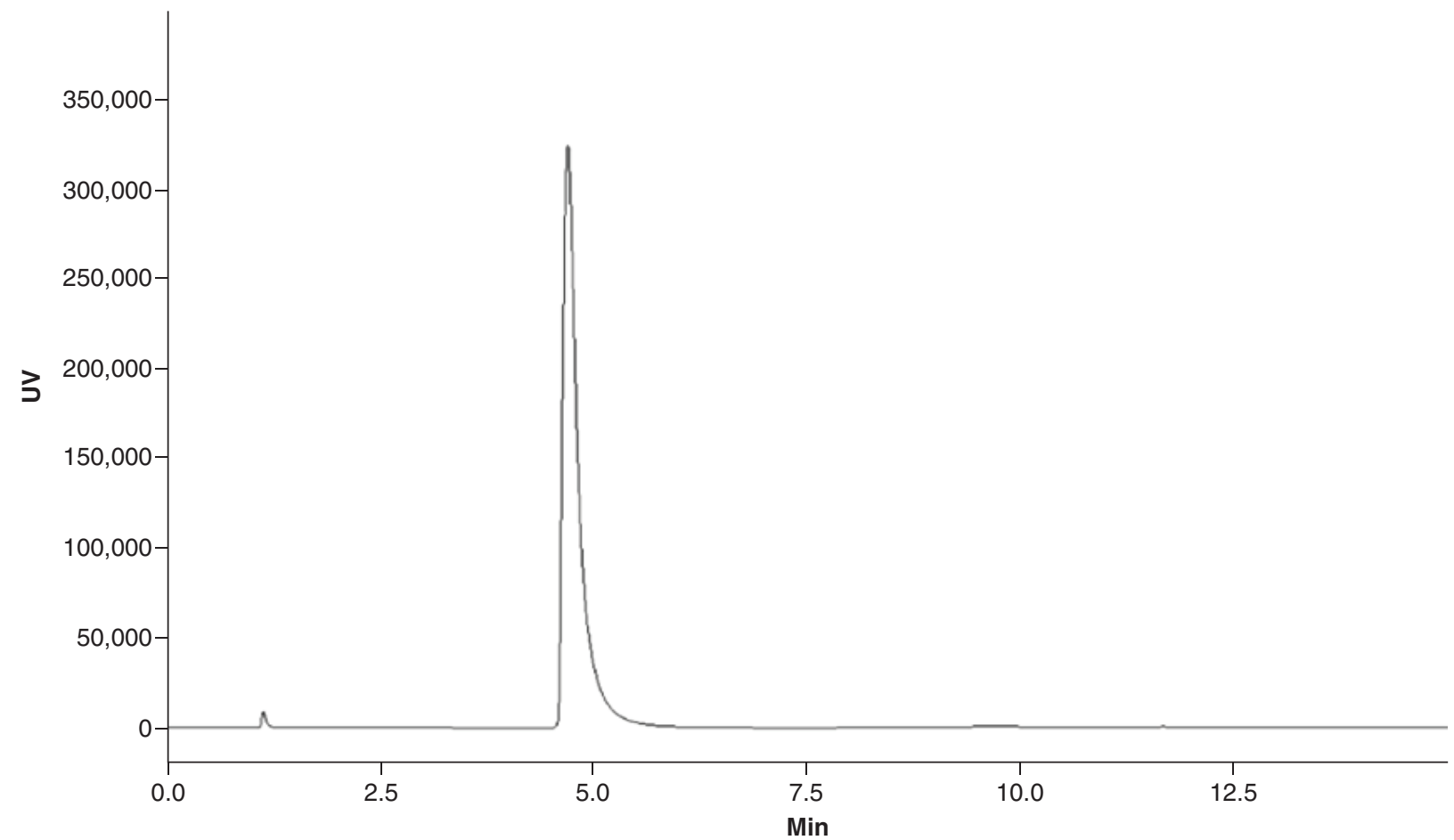

Figure 2. Characterization of refolded and purified IL-17A. (A) SDS-PAGE analysis of the refolded and purified IL-17A in the presence (lane 2) and absence (lane 1) of DTT, corresponding to the monomer and covalent dimer, respectively. (B) RP-HPLC analysis of the purified IL-17A on a Shimazdu LC2030C column (Boston Green C18, $150 \mathrm{~mm} \times 4.6 \mathrm{~mm}, 5 \mu \mathrm{m}$ ). Mobile Phase: A, 0.05\% TFA in water; B, 0.05\% TFA in acetonitrile. Gradient B set from $5 \%$ to $95 \%$ in $10 \mathrm{~min}$ and hold $95 \%$ for $5 \mathrm{~min}$. Flow rate: $1.5 \mathrm{ml} / \mathrm{min}$. Column temperature: $40^{\circ} \mathrm{C}$.

peptide ligands to IL-17A and designed novel macrocyclic ligands that are potent IL-17A antagonists [5,9]. They used both anti-IL-17A Fab and high-affinity peptide (HAP) to stabilize IL-17A and successfully determined $x$-ray structures of the IL-17A/Fab/HAP ternary complex, and the quaternary complexes with the ligands, which have been the only published co-crystal structures of IL-17A with small-molecule compounds so far. However, neither yield nor characterization of the Fab and the complex was reported. In this report, we also address the production of anti-IL-17A Fab with enhanced yield and the characterization of the IL-17A/Fab complex for the first time, aiming to provide a proper assessment of the quality of the IL-17A/Fab complex and hence to obtain the co-crystal structure of IL-17A with smallmolecule antagonists derived from our proprietary DNA-encoded library. 


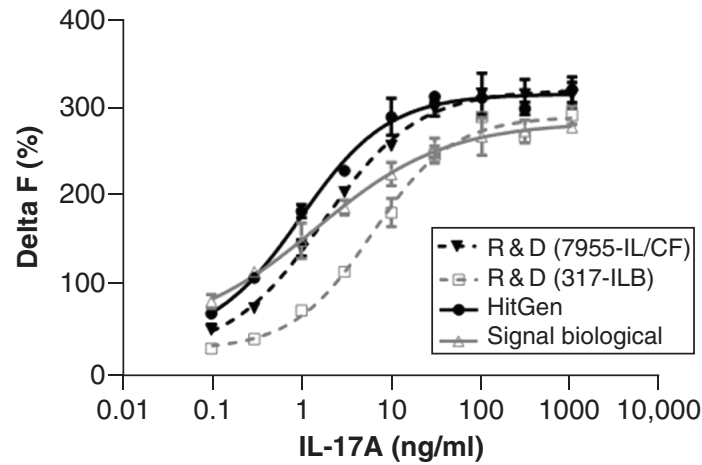

\begin{tabular}{|c|c|c|c|c|}
\hline $\begin{array}{c}\text { IL-17A from } \\
\text { different vendors }\end{array}$ & $\begin{array}{c}\text { Sino biological } \\
(12407-H 07 B)\end{array}$ & $\begin{array}{c}\text { R \& D } \\
(317-I L B)\end{array}$ & $\begin{array}{c}\text { R \& D } \\
(7955-I L / C F)\end{array}$ & $\begin{array}{c}\text { HitGen } \\
\text { (in house) }\end{array}$ \\
\hline Expression system & Insect & E. coli & HEK 293 & E. coli \\
\hline $\mathrm{EC}_{50}(\mathrm{ng} / \mathrm{ml})$ & $1.87 \pm 0.58$ & $5.32 \pm 1.13$ & $1.72 \pm 0.38$ & $0.84 \pm 0.19$ \\
\hline
\end{tabular}

Figure 3. Comparison of IL-17A activity through cell-based cytokine induction assay. The secretion of CXCL1 in HT-29 human colon adenocarcinoma cells stimulated by IL-17A was measured by homogeneous time-resolved fluorescence detection, using a human CXCL1 assay kit (Cat\# 62HCXC1PEG, Cisbio). The final readout was normalized by cell viability. The results were obtained from a representative experiment of two independent experiments, each performed with duplicate samples.

The anti-IL-17A antibody Fab fragment, CAT-2000, with a His6 tag placed at the N-terminus of the heavy chain (HC) was designed as previously described [9]. We placed an Igк leader sequence [17] at the N-terminus of the HC and the light chain (LC), respectively, for protein secretion. The Fab fragment was transiently expressed in human embryonic kidney (HEK) 293-F cells (obtained as a gift from Shanghai Institute of Materia Medica, Chinese Academy of Sciences). The mammalian plasmids encoding Fab HC and LC were mixed at a 1:1 molar ratio and added to the adapted HEK293-F cells at a density of about $2 \times 10^{7}$ cells $/ \mathrm{ml}$ [18]. The transfection reagent, polyethylenimine (Polysciences, Cat\#23966), was added to the cells to a final concentration of $100 \mu \mathrm{g} / \mathrm{ml}$ and mixed gently. The cells were incubated in a shaking incubator for about $4 \mathrm{~h}$, and the culture was diluted 20 -fold in prewarmed SMM-TII/CDM4HEK293 medium. The transient expression gave Fab yields of only around $1.7 \mathrm{mg}$ per liter of cell culture. For the continuous production of larger amounts of Fab protein, a stable 293-F cell line was generated by G418 (Gibco, 10131-035) selection of transfected cells, with a protocol originally designed for another protein, NELL1 - namely, culturing cells with $100 \mu \mathrm{g} / \mathrm{ml}$ of antibiotic G418 for 5 weeks of continuous passage, then maintaining them in medium supplemented with $50-100 \mu \mathrm{g} / \mathrm{ml} \mathrm{G} 418$ [19]. The stable cell line showed enhanced levels of Fab production ( 10-12 mg per liter of cell culture); expression was stable for at least 6 months. Then the cell culture underwent Ni-NTA affinity purification as described previously [9] and the purified Fab were analyzed by nonreducing SDS-PAGE. Since no hinge region was included in the Fab construct, and the fifth cysteine was not included in either the HC or LC of the Fab, the Fab protein formed monomer only and migrated as two bands at around $25 \mathrm{KDa}$ on SDS-PAGE (Figure 5A, lane 1).

The IL-17A/Fab complex was generated as described previously, by mixing the purified IL-17A dimer with slightly more than double the molar ratio amount of anti-IL-17A Fab, then separated from the free Fab by SEC purification on a Superdex 200 column (Figure 4) [9]. The complex exhibited a single symmetric peak with a reasonable retention volume, slightly behind the 158-kDa standard peak of the gel filtration marker (the theoretical molecular weight of the complex is $126 \mathrm{kDa}$ ). The fractions containing the complex were concentrated to $6-7 \mathrm{mg} / \mathrm{ml}$ for crystallization, with the final protein recovery of the complex to be around $50 \%$ with respect to the starting amount of IL-17A for complex formation. A symmetric sharp peak was also observed for the purified complex on SEC-HPLC (data not shown), indicating slow dissociation of the complex. The complex was further characterized by nonreducing and reducing SDS-PAGE (Figure 5B; lanes 3 and 4 show the results in the absence and presence of DTT, with boiling for 3-5 min prior to gel loading. The three sharp bands from top to bottom on lane 3 correspond to the homodimeric IL-17A, Fab HC and LC in the complex. Once reduced, the dimer became monomer ( $15 \mathrm{kDa}$ ), as expected. The Fab HC and LC shifted up slightly, indicating the breaking of disulfide bonds leading to a less compact protein conformation and slower migration on gel. Western blotting with anti-His tag antibody-HRP (CWBIO, Cat\# CW0286) suggested that the $\mathrm{HC}$ shifted up to a lesser degree than LC (the upper, less dense band in lane 4, Figure 5B), but was not detected by the western blotting due to the lack of a tag (Figure 5C). Based on the co-crystallization strategy for IL-17A described by Liu et al. [9], the IL-17A/Fab complex could be successfully co-crystallized with small molecules derived from our proprietary DNA-encoded library, using HAP as a stabilizer. The crystallization conditions were essentially the same but with slight differences: $0.1 \mathrm{M}$ sodium acetate ( $\mathrm{pH} 4.8$ ), 2-6\% (v/v) 2-propanol, 16-28\% (w/v) PEG 6000 and 2\% (w/v) benzamidine hydrochloride using a batch method at $20^{\circ} \mathrm{C}$. The crystals could grow to a size of 100-200 $\mu \mathrm{m}$ on the longest edge within a week; representative co-crystals are shown in Figure 6 . The visible residues start from Thr44, and no interchain disulfides were observed from the resolved co-crystal structures of IL-17A.

\section{Future perspective}

The optimized method for IL-17A refolding and the method for yield enhancement of anti-IL-17A Fab production, as well as characterization of the IL-17A/anti-IL-17A Fab complex, will facilitate future efforts in obtaining IL-17A/small-molecule co-crystals, providing critical 
(A)

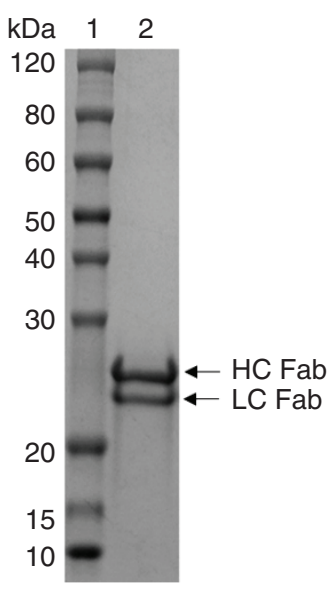

(B)

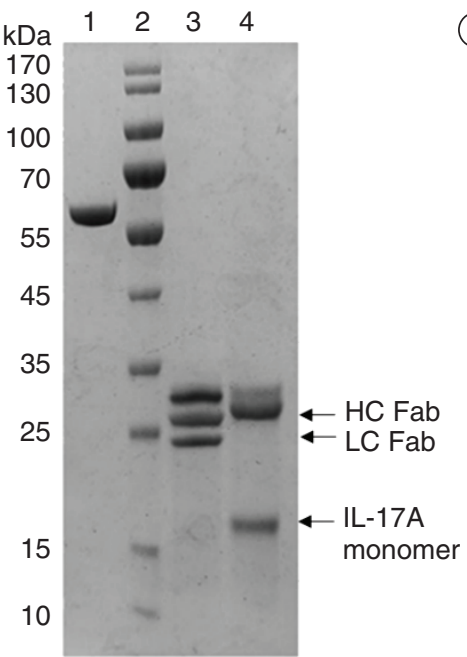

(C)

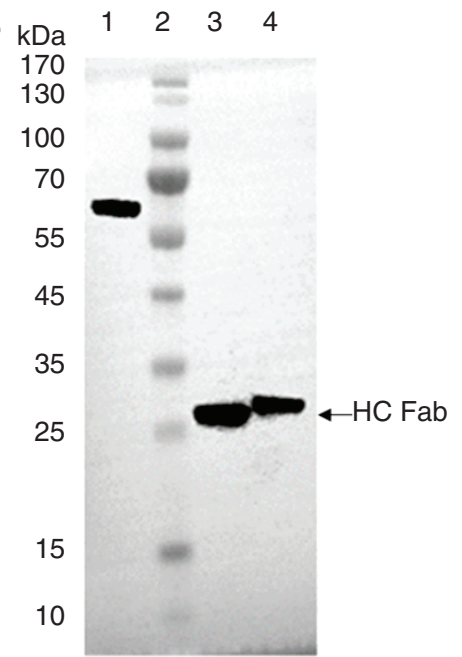

Figure 5. Characterization of the purified anti-IL17A Fab and IL-17A/Fab complex. (A) Lane 1, SDS-PAGE of purified anti-IL-17A Fab with a loading amount of $4 \mu \mathrm{g}$; lane 2, protein standard (PAGE-Master Plus MM1397-500, Genscript). (B) SDS-PAGE and (C) western blotting of IL-17A/Fab complex with anti-His antibody at 1:5000 dilution (HRP anti-His tag antibody, CW0286, CWBIO). Lane 1, His-tagged reference protein as a positive control; lane 3 , the purified IL-17A/Fab complex in the absence of DTT, boiled for $5 \mathrm{~min}$; lane 4, purified IL-17A/Fab complex in the presence of DTT, boiled for 5 min. The voltage setting was at $100 \mathrm{~V}$ for $20 \mathrm{~min}$, followed by $150 \mathrm{~V}$ for $40 \mathrm{~min}$. Protein standard in B and C (Cat\#26617, Thermo Fisher).

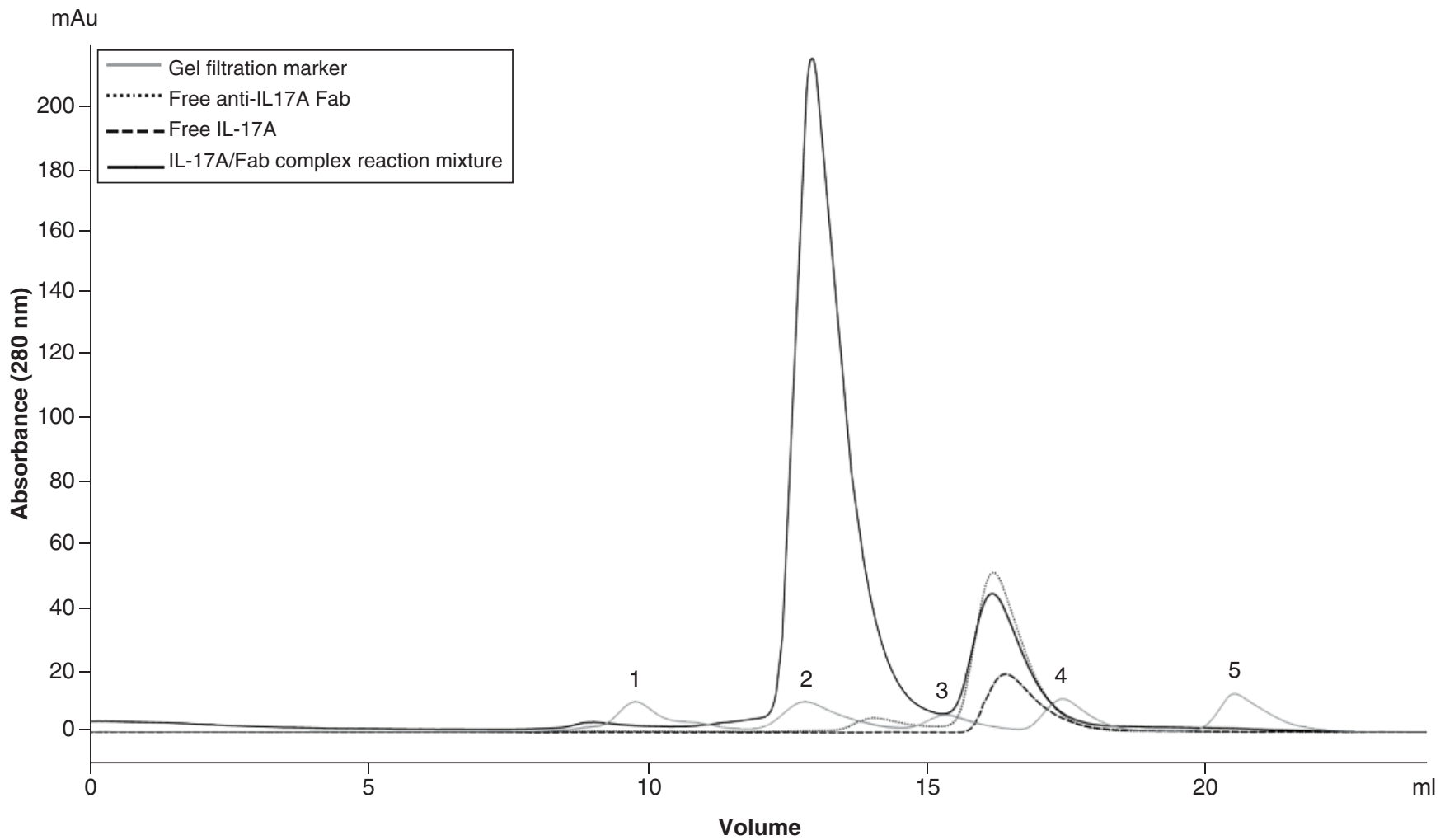

Figure 4. Purification of IL-17A/Fab complex by size exclusion chromatography. The purification was performed on AKTA Pure-25 (GE). The main peak of the reaction mixture eluted at $\sim 13.0 \mathrm{ml}$ corresponds to the complex. The minor peak at $\sim 16.2 \mathrm{ml}$ corresponds to the free Fab in excess amount after the reaction. The standard peaks (1-5) of the gel filtration marker (BioRad, Cat\#151-1901) from large to small correspond to molecular weight of 670, $158,44,17$ and $1.35 \mathrm{kDa}$. 

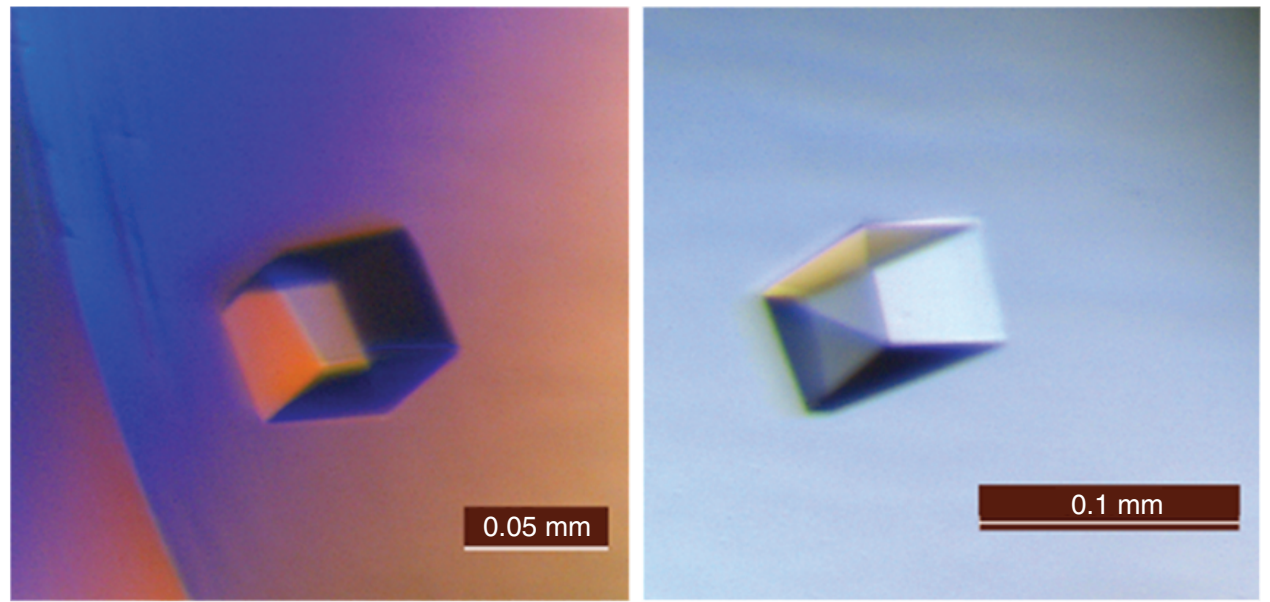

Figure 6. Representative co-crystals of IL-17A quaternary complex with Fab, HAP, and small molecule compounds from DEL.

information for medicinal chemistry lead optimization and structure-based drug design studies on small-molecule drug discovery targeting IL-17A.

\section{Author contributions}

X Meng prepared the manuscript. X Meng and L Zhang designed the study. L Zhang, H Wei, F Li, Q Liu, X Li and H Ma contributed to construct design, protein overexpression, refolding, purification and characterization. $L$ Zhang and $F$ Li contributed to co-crystallization. Z Liu contributed to the x-ray diffraction experiment, data experiment, data collection and analysis. All the authors read and approved the final version of the manuscript.

\section{Acknowledgments}

The authors are grateful to $X$ Zhang and $C$ Yuan for their critical reading of the manuscript, to $L$ Wu for his assistance in the cellular assay for protein bioactivity, and to colleagues from Discovery Chemistry Unit, and Discovery Project Unit for their contributions to the project.

\section{Financial \& competing interests disclosure}

The authors have no relevant affiliations or financial involvement with any organization or entity with a financial interest in or financial conflict with the subject matter or materials discussed in the manuscript. This includes employment, consultancies, honoraria, stock ownership or options, expert testimony, grants or patents received or pending, or royalties.

No writing assistance was utilized in the production of this manuscript.

\section{Open access}

This work is licensed under the Attribution-NonCommercial-NoDerivatives 4.0 Unported License. To view a copy of this license, visit http://creativecommons.org/licenses/by-nc-nd/4.0/

\section{References}

1 Beringer A, Noack M, Miossec P. IL-17 in chronic inflammation: from discovery to targeting. Trends Mol. Med. 22(3), 230-241 (2016).

2 Li X, Bechara R, Zhao J, McGeachy MJ, Gaffen SL. IL-17 receptor-based signaling and implications for disease. Nature Immunol. 20(12), 1594-1602 (2019).

3 Hymowitz SG. IL-17s adopt a cystine knot fold: structure and activity of a novel cytokine, IL-17F, and implications for receptor binding. EMBO J. 20(19), 5332-5341 (2001).

4 Liu S, Song X, Chrunyk BA et al. Crystal structures of interleukin 17A and its complex with IL-17 receptor A. Nat. Commun. 4(1), 1-9 (2013).

5 Liu S, Desharnais J, Sahasrabudhe PV et al. Inhibiting complex IL-17A and IL-17RA interactions with a linear peptide. Sci. Rep. 6(1), 1-11 (2016).

6 Gerhardt S, Abbott WM, Hargreaves D et al. Structure of IL-17A in complex with a potent, fully human neutralizing antibody. J. Mol. Biol. 394(5), 905-921 (2009).

7 Ting JP, Tung F, Antonysamy S et al. Utilization of peptide phage display to investigate hotspots on IL-17A and what it means for drug discovery. PLoS ONE 13(1), (2018).

8 Nimrod G, Fischman S, Austin M et al. Computational design of epitope-specific functional antibodies. Cell Rep. 25(8), 2121-2131 (2018).

9 Liu S, Dakin LA, Xing L et al. Binding site elucidation and structure guided design of macrocyclic IL-17A antagonists. Sci. Rep. 6(1), 1-12 (2016).

10 Wu B, Nemeth JF, Janecki DJ et al. Expression, refolding and purification of a human interleukin-17A variant. Cytokine 53(1), 107-114 (2011).

11 Wu B, Muzammil S, Jones B et al. The role of interchain disulfide bond in a recombinant human Interleukin-17A variant. Cytokine 65(2), 167-174 (2014).

12 Vallejo LF, Rinas U. Strategies for the recovery of active proteins through refolding of bacterial inclusion body proteins. Microbial Cell Factories 3(1), 11 (2004).

13 Wetlaufer DB, Branca PA, Chen GX. The oxidative folding of proteins by disulfide plus thiol does not correlate with redox potential. Protein Eng. Des. Sel. 1(2), 141-146 (1987).

14 Rudolph R, Lilie H. In vitro folding of inclusion body proteins. FASEB J. 10(1), 49-56 (1996).

15 Hassell AM, Wells TN, Graber P et al. Crystallization and preliminary x-ray diffraction studies of recombinant human interleukin-5. J. Mol. Biol. 229(4), 1150-1152 (1993).

16 Kusano S, Kukimoto-Niino M, Hino N et al. Structural basis of interleukin-5 dimer recognition by its $\alpha$ receptor. Protein Sci. 21(6), 850-864 (2012). 
17 Spidel JL, Vaessen B, Chan YY, Grasso L, Kline JB. Rapid high-throughput cloning and stable expression of antibodies in HEK293 cells. J. Immunol. Methods 439, 50-58 (2016).

18 L'Abbé D, Bisson L, Gervais C, Grazzini E, Durocher Y. Transient gene expression in suspension HEK293-EBNA1 cells. In: Recombinant Protein Expression in Mammalian Cells. Humana Press, NY, USA, 1-16 (2018).

19 Hasebe A, Tashima H, Ide T et al. Efficient production and characterization of recombinant human NELL1 protein in human embryonic kidney 293-F cells. Mol. Biotechnol. 51(1), 58-66 (2012) 
